\section{BOLIVIA. DISCURRIENDO SOBRE CUESTIONES LIGADAS A SU POBLACIÓN}

Sonia Elízabeth Jiménez C. ${ }^{1}$

\section{Resumen}

Aproximaciones iniciales a determinados indicadores e informes ligados a la mortalidad, natalidad, fecundidad, educación, emigración, crecimiento anual de la población, así como las miradas retrospectivas al Censo del 2001 y miradas actuales a los resultados del Censo del 2012 otorgan una perspectiva global sobre el estado actual de la población en Bolivia.

\section{BOLIVIA. REFLECTIONS ON MATTERS ABOUT POPULATION}

Sonia Elízabeth Jimenez C. ${ }^{1}$

\section{Abstract}

Initial approaches to certain indicators and reports related to mortality, birth, fertility, education, emigration, annual population growth, a retrospective look at the 2001 Census and reflections on the 2012 Census provide a global perspective on the current state of the population in Bolivia. 
PALABRAS CLAVE: POBLACIÓN; NATALIDAD; FECUNDIDAD; MORTALIDAD; EMIGRACIÓN; EDUCACIÓN; BOLIVIA

Fecha de recepción: 30-09-2013

Fecha de aceptación: 20-01-2015

1 Bolivia. Arquitecta Universidad Mayor de San Simón UMSS de Cochabamba, Magister en Gestión del patrimonio y desarrollo territorial - UMSS. Desarrolla actividades de docencia en el Instituto de Investigaciones de Arquitectura y Ciencias del Hábitat de la Facultad de Arquitectura de la Universidad Mayor de San Simón. Coordinadora del Programa de capacitación para el Mejoramiento Socio-Habitacional - Promesha por el IIACH - UMSS en Bolivia (1999 - 2013). Correo electrónico: soniajimenez777@ gmail.com

\section{KEYWORDS: POPULATION; BIRTH; FERTILITY; MORTALITY; EMIGRATION; EDUCATION; BOLIVIA}

Received: 30-09-2013

Accepted: 20-01-2015

1 Bolivia. BA in Architecture, St. Simon University - UMSS, Cochabamba. MSc in Heritage Management and Territorial Development - UMSS. Professor and researcher at the Research Institute on Architecture and Habitat Sciences, Faculty of Architecture at the St. Simon University. Email: soniajimenez777@ gmail.com 


\section{Miradas preliminares al crecimiento poblacional de Bolivia}

Miradas retrospectivas al crecimiento poblacional de Bolivia (tabla 1) nos llevan a la afirmación de que el crecimiento poblacional en Bolivia ha sido históricamente lento. Bolivia desde su nacimiento a la vida independiente (1825), contaba con una población aproximada de un millón de habitantes. Desde la instauración de la república diversos factores (internos y externos) contribuyeron a retrasar el proceso del crecimiento y desarrollo de Bolivia, particularmente en el periodo intercensal comprendido entre 1854 y 1882, periodo en el que se observa un descenso significativo de la población, atribuible a la aparición de varias epidemias como fiebre intermitente, fiebre tifoidea -suscitadas en el 1878- que elevaron los índices de mortalidad a cifras sin precedentes. A las epidemias, sequías y hambruna generalizada se sumó la guerra del pacifico (1879) que dejó secuelas irreparables en los primeros años de post guerra y abrió paso a una nueva crisis regional que afectó a estratos medios y bajos. Entre los años 1879 y 1880 las migraciones del campo a la ciudad y de la ciudad al campo se intensificaron y más tarde las migraciones intraurbanas entre los años 1897 y 1899. Se conoce que las gentes de la ciudades y las villas provinciales abandonaron las poblaciones para buscar sustento
TABLA 1. BOLIVIA. CRECIMIENTO POBLACIONAL: 1831 - 2012

\begin{tabular}{cc}
\hline Años: $1831-2012$ & Población \\
\hline $\mathbf{1 8 3 1}$ & 1.088 .768 \\
\hline $\mathbf{1 8 3 5}$ & 1.060 .777 \\
\hline $\mathbf{1 8 4 5}$ & 1.378 .896 \\
\hline $\mathbf{1 8 5 4}$ & 1666.126 \\
\hline $\mathbf{1 8 8 2}$ & 1.097 .616 \\
\hline $\mathbf{1 9 0 0}$ & 1.555 .818 \\
\hline $\mathbf{1 9 5 0}$ & 2.704 .165 \\
\hline $\mathbf{1 9 7 6}$ & 4.613 .489 \\
\hline $\mathbf{1 9 9 2}$ & 6.420 .792 \\
\hline $\mathbf{2 0 0 1}$ & 8.274 .325 \\
\hline $\mathbf{2 0 1 2}$ & 10.059 .826 \\
\hline
\end{tabular}

Fuente: INE, 2001

en los campos y al no encontrar esperanzas retornaron a las ciudades flageladas, incapaces de brindar respuestas a las múltiples necesidades de la población citadina y rural.

El primer y segundo censo se realizó bajo la administración del Presidente Andrés de Santa Cruz, en el año 1831 y 1835 respectivamente. El segundo censo llamado "Recuento Poblacional II" señaló como dato oficial, una población de 1.060.777 habitantes. El tercer censo realizado durante el Gobierno de José Ballivián en 1845 arrojó una cifra de 1.378.896 habitantes, de estos 478.000 indígenas 
vivían en comunidades o pueblos de indios. El cuarto censo, realizado bajo la presidencia de Manuel Isidoro Belzu, señaló la cifra de 1.666.126 habitantes, incluidos 100.000 indios salvajes, cuya existencia se suponía en el Beni, Santa Cruz y el Chaco. El Censo de 1882 se realizó bajo la presidencia de Narciso Campero. La reducción de población de ese entonces se atribuyó a las pérdidas territoriales en favor de Chile y Brasil. Además en esa época se suscitaron constantes guerras civiles, pestes y hambrunas que sufrió el país.

Más adelante, en 1950 se realizó el séptimo Censo de Población y Vivienda -el primero de vivienda, bajo el mandato de Mamerto Urriolagoitia- y según datos del referido censo, Bolivia contaba con más de 2.7 millones de habitantes, de los cuales 708.568 residían en áreas urbanas, lo que supuso un incremento de su nivel de urbanización del $26.2 \%$. Diez y seis años después, en 1976, durante el régimen dictatorial de Hugo Banzer Suárez se realizó un nuevo trabajo de relevamiento de datos -el octavo censo nacional de población y segundo de vivienda- el mismo indicaba que el número de habitantes se había duplicado hasta superar los 4.6 millones de habitantes. En el año referido -1976- la mayoría de la población (64.5 por ciento) era indígena originaria, proporción que bajó al 49 por ciento en el 2001. La relativa baja proporción de población urbana, estuvo influenciada por la "reducida capacidad de industrialización de su estructura productiva y la alta proporción de población indígena originaria"2. Dieciséis años después -1992- se realizó el noveno censo de población y tercero de vivienda, durante la gestión gubernamental de Jaime Paz Zamora. Y en este acápite es oportuno abrir un breve paréntesis para explicar que a partir de 1985 "la población urbana logra sobrepasar a su par rural como resultado de procesos sociales, económicos y políticos que lograron transformar a la población boliviana en mayoritariamente urbana"3. De esta manera la tendencia poblacional creciente refiere cambios demográficos asociados a las tasas de natalidad, mortalidad, fecundidad y fundamentalmente a los flujos migratorios campo-ciudad que se suscitaron particularmente entre los años 1950 y 1992, lo que explica el posterior crecimiento poblacional urbano, acentuado en 1985.

Inaugurando el siglo XXI, en el 2001 durante la presidencia de Jorge Quiroga Ramírez se desarrolló el décimo censo de población y vivienda, que arrojó una cifra de 8.274.325 habitantes. Finalmente referimos el último censo que se llevó a cabo en noviembre del 2012 bajo la presidencia de Evo Morales Ayma y de acuerdo con el informe elaborado por el Instituto Nacional de Estadística 
- INE, Bolivia cuenta con 10.059.856 habitantes, es decir, el tamaño de la población se multiplicó cerca de cuatro veces durante un periodo de 62 años, sin embargo, la tasa media de crecimiento anual según departamentos entre el periodo censal 2001 y 2012, refiere una disminución, con excepción de los departamentos de Pando, Oruro y Potosí. El caso de Pando es el más notable, pues su población se duplicó durante los últimos 11 años. La tasa de este departamento se elevó de 3,48 por ciento (intervalo 1992-2001) a 6,63 por ciento (intervalo 2001-2012). En Pando se visualizaba -y aún se visibiliza - un escenario de profundas rupturas y contradicciones similares a las existentes en otros departamentos de Bolivia. En el referido departamento existen problemas serios de comunicación, situación que repercute dramáticamente en la situación de los pandinos, agudizándose los niveles de pobreza, la persistencia de enfermedades endémicas y las malas condiciones de habitabilidad. Situación similar ocurrió con Potosí, que aumentó su tasa anual de crecimiento poblacional de 1,01 por ciento (intervalo 1992 - 2001) a 1,38 por ciento (intervalo 2001- 2012) y Oruro de 1,55 a 2,06 por ciento (en los referidos periodos intercensales). En Oruro los fuertes procesos migratorios internos fueron reestructurando hábitos de comportamiento urbano por la presencia de masas poblacionales de origen rural, lo que ha generado un proceso de ruralización de la ciudad capital. Oruro es un lugar de paso, precisamente porque pervive la idea entre sus habitantes de efectuar inicialmente un ciclo de producción económica y posteriormente realizar traslados a otros departamentos para residir en forma definitiva. La Paz entre 1992 y 2001 refiere una tasa anual de crecimiento del 2.29 por ciento y entre el 2001 y 2012 denota una tasa anual del 1.30 por ciento, cifras que revelan claramente una expulsión de población, mientras que las tasas de Santa Cruz para los mismos periodos son de 4.29 y 2.41 por ciento respectivamente, lo que explica por qué este último departamento casi supera en población al departamento de La Paz. De esta manera Bolivia registró en un intervalo de 11 años - después del censo del 2001- un incrementó de 1.785.531 habitantes.

$\mathrm{Al}$ presente Bolivia tiene una tasa anual de crecimiento poblacional de 1,74 menor al 2,74 que reportó el empadronamiento del 2001 en un intervalo de nueve años (1992-2001), correspondiendo al área urbana una tasa anual de crecimiento del 3.62 por ciento y al área rural del 1.42 por ciento. Declinación poblacional -a decir de expertos demográficos- acorde con la desaceleración paulatina del crecimiento de la población Latino Americana, que "después de triplicarse entre 1950 y 2010, al pasar de 167 a 590 millones de habitantes, se prevé que hasta 2030 Latinoamérica registre una 
subida del 20 por ciento y sólo un 9 por ciento más hacia el año 2050"4.

Es conveniente puntualizar que Bolivia, a pesar de contar con uno de los más bajos niveles de urbanización de América Latina, en el último cuarto de siglo (1980 - 2005), la "proporción de su población urbana (constituido por los centros poblados con más de 2000 habitantes), se incrementó en veintidós puntos porcentuales, del $44.5 \%$ al $66.5 \%$, lo que demuestra que su crecimiento urbano fue visiblemente superior al promedio de América Latina, por tanto, se convierte en uno los países con mayor crecimiento urbano de la región"5.

\section{Bolivia. Matices diversos sobre su población}

Bolivia es un país con un desarrollo humano medio, que se refleja en el estado de los asentamientos humanos y de la vivienda en particular. Ocupa el puesto 108 entre 187 países según los Índices de Desarrollo Humano del Programa de las Naciones Unidas para el Desarrollo ${ }^{6}$, índices sujetos a una clasificación que mide indicadores nacionales de salud, educación e ingresos a nivel mundial (tabla 2).

\footnotetext{
Latinos viven más años (...) 2013, p. 10.

CEPAL, 2005.

PNUD, 2013, p. 2.
}

El valor del Índice de Desarrollo Humano del país ha aumentado de 0,671 a 0,675 entre 2011 y 2012, según información proveniente del PNUD 2013, debido principalmente al incremento del 3 por ciento en el ingreso per cápita entre esos dos años. Las tendencias del índice de desarrollo humano, en base a información proveniente de la misma fuente, refieren que desde 1980 el progreso del país fue sustancial y sostenido. Hace 33 años, los bolivianos gozaban de una esperanza de vida de 52 años, una escolaridad promedia de 4,5 años y un ingreso per cápita de 3.791 dólares Americanos (calculado en dólares constantes del año 2005). Hoy en día, la esperanza de vida al nacer es de casi 67 años, la escolaridad promedia es de 9,2 años y el ingreso per cápita es 4.444 dólares americanos. De esa manera entre los años 1980 y 2012, el Índice de Desarrollo Humano subió de 0,489 a 0,675 (tabla 3), sin embargo, el progreso en la esfera económica ha sido más bien modesto y "nuestros niveles de desigualdad en la repartición del ingreso, medidos por el coeficiente de Gini (56,3 en Bolivia), siguen siendo unos de los peores existentes a nivel regional y mundial, comparables únicamente con países del continente como Colombia $(55,9)$, Honduras $(57,0)$, Guatemala $(55,9)$, Haití $(59,2)$ y Brasil $(54,7)$, así como con naciones 
TABLA 2. ÍNDICES DE DESARROLLO HUMANO EN AMÉRICA LATINA - 2013

\begin{tabular}{|c|c|c|c|}
\hline \multicolumn{4}{|c|}{$\begin{array}{c}\text { ÍDH } 2013 \\
\text { Países seleccionados de América Latina }\end{array}$} \\
\hline Clasificaciór & 013 & IDH 20 & IDH 2012 (Valor) \\
\hline \multicolumn{4}{|c|}{ Desarrollo humano muy alto } \\
\hline Chile & 40 & 0.817 & 0.819 \\
\hline Argentina & 45 & 0.810 & 0.811 \\
\hline \multicolumn{4}{|c|}{ Desarrollo humano alto } \\
\hline Uruguay & 51 & 0.789 & 0.792 \\
\hline Venezuela & 71 & 0.746 & 0.748 \\
\hline Perú & 77 & 0.738 & 0.741 \\
\hline Brasil & 85 & 0.728 & 0.730 \\
\hline Ecuador & 89 & 0.722 & 0.724 \\
\hline Colombia & 91 & 0.717 & 0.719 \\
\hline \multicolumn{4}{|c|}{ Desarrollo humano medio } \\
\hline El Salvador & 107 & 0.679 & 0.680 \\
\hline Bolivia & 108 & 0.671 & 0.675 \\
\hline Paraguay & 111 & 0.670 & 0.669 \\
\hline Honduras & 120 & 0.630 & 0.632 \\
\hline Nicaragua & 129 & 0.597 & 0.599 \\
\hline Guatemala & 133 & 0.580 & 0.581 \\
\hline
\end{tabular}

Fuente: PNUD, 2013 


\section{TABLA 3. TENDENCIAS DEL ÍNDICE DE DESARROLLO HUMANO EN BOLIVIA}

\begin{tabular}{lcccc} 
Años & $\begin{array}{c}\text { Esperanza de vida al nacer } \\
\text { (años) }\end{array}$ & Promedio de escolaridad (años) & INB Per cápita (\$us PPA 2005) & IDH \\
1980 & 52.0 & 4.5 & 3.791 & 0.489 \\
\hline 1990 & 55.8 & 6.4 & 2.922 & 0.557 \\
\hline 2000 & 63.0 & 7.4 & 3.472 & 0.620 \\
\hline 2010 & 66.3 & 9.2 & 4.163 & 0.668 \\
\hline 2011 & 66.6 & 9.2 & 4.315 & 0.671 \\
\hline 2012 & 66.9 & 9.2 & 4.444 & 0.675 \\
\hline
\end{tabular}

Fuente: PNUD, 2013

africanas como Sudáfrica $(63,1)$, Namibia $(63,9)$, Angola $(58,6)$, Zambia $(54,6)$ y Ruanda $(53,1)$ "'7.

En la actualidad Bolivia cuenta con 10.059.856 habitantes, según los últimos datos oficiales del Censo Nacional de Población y Vivienda 2012. La población distribuida por departamentos no es homogénea. El departamento con mayor población es La Paz con 2.719.344 personas, le sucede Santa Cruz con 2.657 .762 personas y Cochabamba que ocupa el tercer lugar con 1.762 .761 personas. La mayor parte de la población se encuentra concentrada en las ciudades del eje principal, Cochabamba, La Paz y Santa Cruz (figura 1).

Al presente el país se encuentra en un proceso acelerado de urbanización, como resultado de las

PNUD, 2013, p. 1. migraciones del campo hacia los centros poblados. Las razones del éxodo de una gran cantidad de población rural, se explican por la ausencia de facilidades de producción y trabajo lo que obliga a la gente a buscar mejores expectativas de vida en las ciudades e incorporarse como mano de obra barata y oficios de cuenta propia mal remunerados. Situación que nos hace entrever que hay una estructura productiva baja, terciarizada y por ende un desarrollo económico inequitativo e insustentable.

Asimismo, es importante señalar que de las 74 poblaciones bolivianas con más de 20.000 habitantes, 19 poblaciones intermedias están en Cochabamba, por consiguiente, según el Censo 2012, Cochabamba es uno de los departamentos con 
FIGURA 1. BOLIVIA, POBLACIÓN POR DEPARTAMENTOS, CENSOS 2001 - 2012

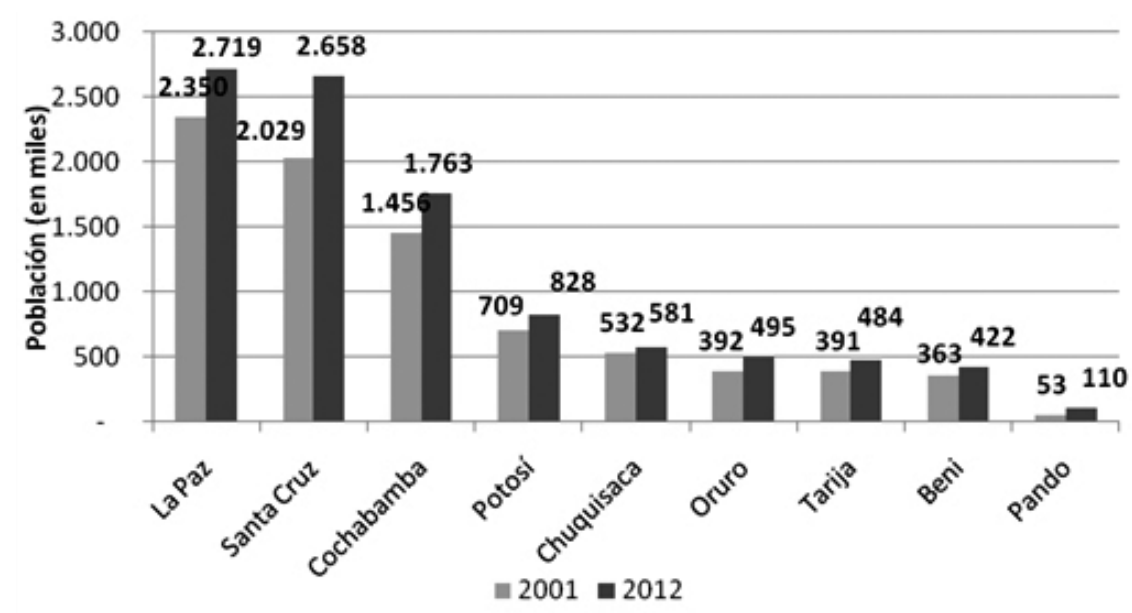

Fuente: INE, 2012

más ciudades intermedias. El departamento de Santa Cruz tiene 16, Potosí posee 13 y La Paz 12 poblaciones intermedias. En el departamento de Tarija, cinco ciudades tienen más de 20.000 habitantes, Beni cuenta con cuatro ciudades intermedias, Oruro con tres y Chuquisaca con dos. En el año 2001 había únicamente 20 ciudades intermedias $^{8}$. El aumento de ciudades intermedias en el país refiere el rápido crecimiento urbano, tres de cuatro ciudadanos conforman la población urbana.

Hoy Bolivia tiene una densidad poblacional de 9.3 hab/km2. En el 2001, el país registraba una densidad de $7.7 \mathrm{hab} / \mathrm{km} 2$, sin embargo y a pesar del leve incremento actual -de $1.6 \mathrm{hab} / \mathrm{km} 2-$ Bolivia ocupa el último lugar de 20 países que conforman América Latina y el Caribe, Argentina tiene 15 
TABLA 4. BOLIVIA: POBLACIÓN DEPARTAMENTAL, SUPERFICIE Y DENSIDAD DE POBLACIÓN. CENSOS 2001 2012

\begin{tabular}{cccccc} 
Departamento & $\begin{array}{c}\text { Superficie } \\
(\mathrm{Km} 2)\end{array}$ & $\begin{array}{c}\text { Población } \\
\text { Censo 2001 }\end{array}$ & $\begin{array}{c}\text { Densidad } \\
\text { hab/km2. }\end{array}$ & $\begin{array}{c}\text { Población } \\
\text { Censo 2012 }\end{array}$ & $\begin{array}{c}\text { Densidad } \\
\text { hab/km2. }\end{array}$ \\
\hline TOTAL & 1.098 .581 & 8.274 .325 & 7.7 & 10.059 .856 & 9.3 \\
\hline Chuquisaca & 51.524 & 531.522 & 10.3 & 581.347 & 11.3 \\
\hline La Paz & 133.985 & 2.349 .885 & 18.0 & 2.719 .344 & 20.9 \\
\hline Cochabamba & 55.631 & 1.455 .711 & 26.2 & 1.762 .761 & 31.7 \\
\hline Oruro & 53.588 & 392.451 & 7.9 & 494.587 & 9.9 \\
\hline Potosí & 118.218 & 709.013 & 6.6 & 828.093 & 7.7 \\
\hline Tarija & 37.623 & 391.226 & 10.4 & 483.518 & 12.9 \\
\hline Santa Cruz & 370.621 & 2.029 .471 & 5.5 & 2.657 .762 & 7.2 \\
\hline Beni & 213.564 & 362.521 & 1.7 & 422.008 & 2.0 \\
\hline Pando & 63.827 & 52.525 & 0.8 & 110.436 & 1.7 \\
\hline
\end{tabular}

Fuente: INE - Censo 2012

habitantes por Km2, Paraguay 17 y otros países limítrofes 23 habitantes por Km2.

En la actualidad los departamentos que tienen mayor densidad poblacional son Cochabamba (31.7 hab/km2), La Paz (20.9 hab/km2) y Tarija (12.9 hab/km2) y los departamentos con menor densidad poblacional son Beni con 2.0 hab/km2 y Pando con 1.7 hab/km2 (tabla 4). En los departamentos con mayor densidad poblacional es posible inferir que el acceso al suelo habitable es cada vez más difícil respecto a otros departamentos menos poblados como Pando y Beni, donde existe mayor suelo para producir y habitar.
Las cifras referidas en la tabla 4 -Censo 2012nos llevan a colegir la presencia de dos grupos de densidad poblacional, el primer grupo con mayor densidad correspondería a los departamentos de Cochabamba (31.7), La Paz (20.9), Tarija $(12,9)$, Chuquisaca $(11,3)$ y Oruro $(9,9)$. El segundo grupo con bajos niveles de densidad correspondería a Potosí (7.7), Santa Cruz (7,2), Beni (2.0) y Pando con $1,7 \mathrm{hab} / \mathrm{km} 2$. Si analizamos los datos provenientes del Censo del 2001, observamos que no hay cambios sustanciales y los distintos departamentos del país responden a la categorización señalada, a excepción del departamento de Pando que de una 
TABLA 5. BOLIVIA: TASAS MEDIAS DE CRECIMIENTO ANUAL DE LA POBLACIÓN SEGÚN DEPARTAMENTOS. CENSOS 1992 - 2012

\begin{tabular}{ccc} 
Departamentos & Periodo: 1992 - 2001 & Periodo: 2001 - 2012 \\
TOTAL & 2.74 & 1.74 \\
Chuquisaca & 1.71 & 0.80 \\
\hline La Paz & 2.29 & 1.30 \\
\hline Cochabamba & 2.93 & 1.71 \\
\hline Oruro & 1.55 & 2.06 \\
\hline Potosí & 1.01 & 1.38 \\
\hline Tarija & 3.18 & 1.89 \\
\hline Santa Cruz & 4.29 & 2.41 \\
\hline Beni & 2.94 & 1.36 \\
\hline Pando & 3.48 & 6.63
\end{tabular}

Fuente: INE, 2012.

densidad poblacional de $0.8 \mathrm{hab} / \mathrm{km} 2$ (censo 2001) pasó a 1.7 hab/km2, según el Censo 2012, lo que nos lleva a percibir un incremento importante de la densidad poblacional en los últimos once años superior al 100 por ciento. Aumento atribuible a la política de re poblamiento promovida el 2009 por el Gobierno de Evo Morales, de esa manera numerosos campesinos del valle de Cochabamba, de los andes: La Paz, Oruro y Potosí, así como zafreros del Beni fueron trasladados al norte amazónico de Pando para conformar los primeros asentamientos comunitarios.
Miradas a las tasas medias de crecimiento anual de la población según departamentos (tabla 5), nos llevan a la conclusión de que en la actualidad en la mayoría de los departamentos de Bolivia existe una desaceleración del crecimiento poblacional. Al respecto diversos expertos del tema señalan que esa desaceleración se atribuye a tres factores dinámicos: mortalidad (que afecta particularmente a la población infantil de las áreas rurales), emigración internacional (éxodo masivo de habitantes hacia países limítrofes y otros continentes) o disminución de la fecundidad. 
Los datos censales en cuanto a la evolución de la tasa de mortalidad en Bolivia en los últimos años, nos llevan a la visualización de un descenso de la misma, por cuanto en el 2001 la mortalidad alcanzaba el 8.29 por ciento y en el 2012 se registra el 7.15 por ciento. Y en este acápite es pertinente referir el Informe global de desarrollo humano 2013, realizado por el Programa de las Naciones Unidas respecto a la salud, el mismo refería que el Estado Plurinacional de Bolivia en el 2010 tenía una tasa de mortalidad infantil de 42 niños por cada mil nacidos vivos y de 54 niños menores de cinco años. En el año 2009 las tasas de mortalidad de mujeres alcanzaba la cifra de 132 mujeres y 203 hombres. En cuanto a la calidad de la atención de la salud la misma fuente señala que el $50 \%$ de los encuestados entre el periodo 2007-2009 señalaron satisfacción con la calidad de la atención de la salud 9 .

La información obtenida en el Censo 2012 refiere que "el 69.2 por ciento de los partos, a nivel nacional, fueron atendidos en establecimientos de salud; esto representa un incremento del 16 por ciento en los últimos once años. En el área rural, la asistencia a un establecimiento de salud se duplicó, es decir, que si bien en el 2001 asistían 20 mujeres de cada 100 a dichos establecimientos, en el último

$9 \quad$ PNUD, 2013, p. 4. censo, esta proporción se incrementó a 40 mujeres por cada cien"10.

La inexistencia de datos e información precisa y actualizada que permita revisar la mortalidad materna e infantil; vacunas a niños hasta un año; número de personas con VIH/SIDA; gasto público en salud; esperanza de vida al nacer; registros cuantitativos de diversas enfermedades como la tuberculosis, Chagas y otras; así como los recursos físicos de salud disponibles por habitante; la ausencia de monitoreos adecuados del derecho a la salud, no permiten la visualización del estado real situacional de la salud en Bolivia. De esta manera diversos organismos como la ONU califican el cumplimiento del derecho a la salud como "cumplimiento moderado grave", lo que nos lleva a entrever que en realidad no se está cumpliendo de manera efectiva el derecho humano a la salud.

Respecto a la fecundidad el 27 de mayo del 2013, el periódico "La Razón" informaba que el promedio de hijos por madre en el país se redujo del 6,7 en 1960 al 3,3 en el 2011. Asimismo el Fondo de Población de las Naciones Unidas -UNFPA- alertó que Bolivia registraba una de las tasas más altas de embarazo adolescente de América Latina y el Caribe. "El promedio de la fecundidad adolescente de América Latina y el Caribe es de 70 nacimientos por cada mil mujeres con edades entre 15 y 19

10 INE, 2012. 
años, pero en Bolivia esa tasa es de 88. En Bolivia tres de cada diez adolescentes del grupo más pobre es madre o está embarazada frente a una de cada diez del sector más rico" (La Paz, EFE, julio 2013). Los datos son sugestivos y denotan que el promedio de fecundidad de adolescentes es elevado, sin embargo, los embarazos son interrumpidos, de esta manera Bolivia es uno de los países con más altas tasas de aborto del continente latinoamericano y así corroboran los estudios realizados por UNFPA, los mismos señalan que cada año interrumpen su embarazo entre 40.000 y 80.000 mujeres con un promedio de 115 casos diarios. Además 3 de cada 5 bolivianas se somete a un aborto alguna vez en su vida. Los diversos informes y cifras referidos son altamente preocupantes y nos llevan a reflexionar sobre lo que se viene haciendo en materia de salud pública y educación sexual y nos inducen a pensar en la búsqueda de soluciones integrales direccionadas a combatir efectivamente las elevadas tasas de embarazos de adolescentes.

Datos e informes diversos nos llevan a la afirmación de que el derecho a la salud no ha logrado su plenitud y a manifestar la necesidad de rediseñar nuevos mecanismos de políticas públicas de salud, mejorar la calidad de los servicios de salud y atención, crear nuevos marcos de coordinación institucional a nivel nacional y regional, así como nuevos mecanismos de control social. Por consiguiente, es posible sostener que las brechas existentes en el campo de la salud refieren atraso de políticas, programas y proyectos de salud que afectan particularmente a los sectores más vulnerables y que ponen en cuestionamiento los preceptos básicos de la actual Constitución Política del Estado Plurinacional Boliviano ${ }^{11}$.

Al presente se aguarda la efectivización de los derechos de salud y seguridad social pregonados en la actual Constitución, vía reglamentaciones y normas complementarias, ya que si bien las condiciones en general han mejorado, el cumplimiento del derecho a la salud, queda cuestionado, debido a que aún no es universal y de acceso a todos los bolivianos sin excepción. Urge una revisión y diagnóstico situacional, así como acciones de corto, mediano y largo plazo en la perspectiva del logro efectivo del derecho a la salud. El reto para el actual gobierno es garantizar la equidad en el servicio de salud y evitar la provisión de servicios de mala calidad direccionados a los pobres esencialmente. "Pueden diseñarse e implementarse políticas de salud pública universales de una manera que no sacrifique la calidad, en beneficio de una cobertura más amplia ${ }^{12}$. Por consiguiente, es improrrogable la ampliación de la cobertura de los

11 Bolivia, 2009.

12 PNUD, 2013, p. 82. 
TABLA 6. BOLIVIA. EMIGRACIÓN INTERNACIONAL

\begin{tabular}{ccccccccccc}
\multicolumn{8}{c}{ Personas que emigraron por departamento en el momento del Censo 2012} \\
$\begin{array}{c}\text { Total } \\
\text { emigrantes }\end{array}$ & Chuquisaca & La Paz & Cbba. & Oruro & Potosí & Tarija & Santa & Beni & Pando \\
\hline 562.461 & 32.943 & 94.632 & 127.441 & 24.777 & 131.441 & 24.147 & 112.183 & 13.470 & 1.427 \\
\hline
\end{tabular}

Fuente: INE, 2012

servicios de salud en un marco de integralidad y sostenibilidad.

Visualicemos otro indicador relevante, la emigración internacional acentuada en los últimos años. El Censo 2012 registró la salida de 562.461 personas a otros países durante los últimos 11 años, es decir, en el periodo 2001 - 2012 (tabla 6). Del total de 562.461 migrantes que registró el Censo del 2012, el departamento con mayor emigración es Potosí con 131.441 personas que representan el 23.4 por ciento, luego está el departamento de Cochabamba con 127.441 personas, equivalentes al 22.6 por ciento y el departamento de Santa Cruz con 112.183 personas, equivalentes al 19.94 por ciento del total de migrantes.

Según el Censo 2012 los países de mayor recepción de migrantes son: Argentina con 228.082 personas, España con 113.628 personas, Brasil con 54.417 personas, y Chile con 31.313 personas. La mayoría de los migrantes bolivianos, más de 300.000 bolivianos están entre 15 y 29 años de edad, la mitad de estos están entre los 20 y 24 años, de los cuales, 66.000 son varones y 65.000 son mujeres.

En los últimos años la emigración se ha constituido en una respuesta a la precarización generalizada de empleo de la población boliviana y devela pobreza y exclusión, situación que refiere la ineficiencia de políticas económicas y sociales que soslayan a las personas que no tienen capacidad productiva. Como resultado de esta segregación cada vez son más la personas que emigran con la ilusión de encontrar en otros países trabajo aunque se conoce que muchos migrantes sufren discriminación y sus posibilidades de inserción laboral son mínimas o nulas lo que acrecienta su vulnerabilidad.

En cuanto concierne a la educación, es posible visualizar el indicador de alfabetización. En la actualidad se conoce que existe una cobertura de alfabetización que llega al 94.98 por ciento a nivel nacional, es decir 6.899.308 de personas 
TABLA 7. BOLIVIA. TASA DE ALFABETISMO DE LA POBLACIÓN DE 15 AÑOS 0 MÁS DE EDAD POR CENSO Y SEXO, SEGÚN DEPARTAMENTO (EN PORCENTAJES). CENSOS: 2001 - 2012

\begin{tabular}{|c|c|c|c|c|c|c|c|c|}
\hline \multirow[t]{2}{*}{ Departamento } & \multirow[t]{2}{*}{ TOTAL } & \multicolumn{3}{|c|}{ CENSO 2001} & \multirow[t]{2}{*}{ TOTAL } & \multicolumn{3}{|c|}{ CENSO 2012} \\
\hline & & Hombres & Mujeres & $\begin{array}{l}\text { Diferencia hombre } \\
\text { - mujer }\end{array}$ & & Hombres & Mujeres & $\begin{array}{l}\text { Diferencia hombre } \\
\text { - mujer }\end{array}$ \\
\hline TOTAL & 86,72 & 93,06 & 80,65 & 12,41 & 94,98 & 97,49 & 92,54 & 4,94 \\
\hline Chuquisaca & 73,03 & 81,62 & 65,21 & 16,41 & 88,98 & 93,35 & 84,86 & 8,49 \\
\hline La Paz & 88,61 & 95,15 & 82,44 & 12,71 & 95,37 & 97,66 & 93,16 & 4,5 \\
\hline Cochabamba & 85,47 & 92,58 & 78,84 & 13,74 & 94,38 & 97,58 & 91,39 & 6,19 \\
\hline Oruro & 89,39 & 96,42 & 82,71 & 13,71 & 96,1 & 98,8 & 93,57 & 5,23 \\
\hline Potosí & 71,58 & 84,64 & 60,03 & 24,61 & 89,19 & 95,08 & 83,62 & 11,46 \\
\hline Tarija & 85,9 & 92,09 & 79,91 & 12,18 & 94,37 & 96,96 & 91,86 & 5,1 \\
\hline Santa Cruz & 92,74 & 95,74 & 89,74 & 6 & 97,48 & 98,56 & 96,39 & 2,17 \\
\hline Beni & 91,12 & 93,82 & 88,14 & 5,68 & 96,9 & 97,8 & 95,94 & 1,86 \\
\hline Pando & 89,63 & 92,11 & 86,25 & 5,86 & 97,69 & 98,3 & 96,94 & 1,36 \\
\hline
\end{tabular}

Fuente: INE, 2012

alfabetizadas, según el Censo 2012. El censo del 2001 registraba un total de personas alfabetizadas de 5.047.139 personas alfabetizadas, es decir, un 86.72 por ciento (tabla 7 ). Al respecto recordemos que en el año 2009 Bolivia fue declarada como el tercer país en Latinoamérica libre de analfabetismo, situación que se revierte con los últimos datos del Censo 2012. En el 2009 se contaba con un 3.77 por ciento de analfabetos y en la actualidad la cifra se ha incrementado a 5.02 por ciento. Situación que es atribuible a la presencia de analfabetos funcionales (personas que aun sabiendo leer y escribir no saben aplicar sus conocimientos). Por otra parte el Censo 2012 refiere un incremento importante de la tasa de alfabetismo de las mujeres (92.54) respecto a la tasa de hombres (97.49). De acuerdo al Censo del 2001, la tasa de alfabetismo de las mujeres llegaba a 80.65 y la de hombres a 93.06 , como se indica en la tabla 7 .

Según el Censo 2012, los departamentos de La Paz, Oruro, Santa Cruz, Beni y Pando registran tasas de alfabetismo superiores al promedio nacional de 94.98 por ciento, sin embargo Chuquisaca, Cochabamba, Potosí y Tarija presentan tasas inferiores. 
Los departamentos de Pando y Santa Cruz registran mayores niveles de alfabetismo (97.69 y 97.48 por ciento respectivamente); mientras Chuquisaca registra el menor nivel con 88.98 por ciento de personas de 15 años o más que saben leer y escribir. Según los dos últimos censos (2001 y 2012) el departamento que presenta mayor aumento del nivel de alfabetismo es Potosí, que de 71.58 por ciento en el 2001 pasó al 89.19 por ciento en el 2012, con un incremento de 17.61 puntos porcentuales, seguido del departamento de Chuquisaca que registraba 73.03 por ciento en el 2001 y aumentó a 88.98 por ciento en el 2012, con un incremento de 15.95 puntos. Mientras que el departamento con menor incremento es Santa Cruz, ya que del 92.74 por ciento en el 2001 pasó a 97.48 por ciento en el 2012, con diferencia de 4.74 puntos $^{13}$.

Sobre la tasa de asistencia escolar el Censo 2012 revela que la tasa de asistencia de la población en edad escolar en general alcanza a 83.54 por ciento, mientras que en el 2001 llegó a 79.71 por ciento, lo que significa un incremento del 3.83 por ciento frente a las cifras de hace una década y 72.32 por ciento en 1992 (observándose una subida de 11. 22 por ciento en los últimos 20 años). Otro dato importante es la mayor asistencia de la población femenina en edad escolar a los colegios del país. El informe refiere que en las dos décadas que separan a los censos de 1992 y 2012 la cantidad de niñas

13 INE, 2012, p. 12. que asisten a la escuela subió en un 13.5 por ciento. El incremento de estudiantes de sexo femenino desde el 2001 fue del 5.13 por ciento.

Estas cifras y otras nos llevan a colegir que en la actualidad, a pesar de los avances realizados en materia educativa y disminución significativa del índice de analfabetismo entre los años 2001 y 2012, persisten desigualdades importantes entre los dos sexos. Las mujeres históricamente han sufrido mayores tasas de analfabetismo y de deserción escolar, así como menores niveles de instrucción respecto a la población masculina. Un informe sobre Bolivia publicado por la CEDAW el 2008, sostenía que estas diferencias eran atribuibles a "la falta de infraestructura, a las distancias, al riesgo de sufrir violencia, al costo del transporte y al idioma, factores que comprometen la educación de las niñas y mujeres de las zonas rurales e indígenas del país"14. Aún persisten brechas educativas que ubican a las mujeres en situación de franca desventaja, de esta manera es posible sostener que las desigualdades educativas que sufren hombres y mujeres en Bolivia, son graves ya que la instrucción deficiente o nula tiene secuelas negativas -particularmente en las mujeres- por cuanto determinan sus condiciones de trabajo e ingresos económicos, sus condiciones de habitabilidad, sus relaciones familiares y de pareja, exponiéndolas a violencia doméstica. En síntesis es posible es posible sostener que la carrera

14 Cedaw, 2008, p. 7. 
educativa de los bolivianos y bolivianas está llena de tropiezos y obstáculos que están en relación directa con el género, la edad, la ubicación o lugar de residencia, condición económica, oportunidades, valores y cultura

Finalmente es oportuno rememorar que la educación es un derecho fundamental de las personas y está sustentada y respaldada por múltiples instrumentos internacionales, entre ellos la Declaración Universal de los Derechos Humanos de 1948 y el artículo 17 de la actual Constitución Política del Estado Plurinacional de Bolivia que sostiene: "Toda persona tiene derecho a recibir educación en todos los niveles de manera universal, productiva, gratuita, integral e intercultural, sin discriminación". A su vez el artículo 77 refiere: "La educación constituye una función suprema y primera responsabilidad financiera del Estado, que tiene la obligación indeclinable de sostenerla, garantizarla y gestionarla". La educación ciertamente es el camino más acertado para romper el círculo vicioso de la pobreza y sus lacerantes secuelas, por consiguiente, requiere de políticas y mecanismos efectivos conducentes a su transformación.

\section{Consideraciones finales}

Bolivia históricamente ha tenido un ritmo lento de crecimiento poblacional y en los últimos años -como refiere el Censo 2012- se observa una desaceleración del mismo. Si bien es evidente que el decrecimiento poblacional puede ser atribuible a factores de orden interno y externo, no es menos evidente que un crecimiento demográfico en condiciones óptimas depende de la organización social y de la transformación de la economía que comporta a su vez diversificación de la industria y aprovechamiento racional de los recursos naturales.

En Bolivia, a pesar de los cambios suscitados en los últimos años aún prevalecen dualidades importantes entre ciudades más densamente pobladas y menos pobladas, entre ciudadanos ricos y pobres, lo que nos lleva a visualizar poblaciones heterogéneas con francas dicotomías y asimetrías. En la actualidad la población boliviana se debate en medio de la persistencia de viejos y arraigados problemas como la salud, educación, pobreza, desigualdad y exclusión que afectan a gran parte de la población boliviana.

El escenario descrito a partir de miradas retrospectivas al Censo del 2001 y miradas actuales a determinados indicadores del Censo 2012, brindan una perspectiva global y matices diversos sobre la población boliviana, invitándonos a reflexionar sobre la necesidad de transformación del entorno con una perspectiva social sustentada en una convivencia armónica, de afirmaciones, críticas y libertades dirigidas a promover un crecimiento poblacional inclusivo y equitativo. 


\section{Bibliografía}

BOLIVIA. Constitución política del Estado. La Paz, Bolivia, Editorial UPS. 2009.

CEDAW Comité para la Eliminación de la Discriminación Contra la Mujer. Observaciones finales del Comité para la Eliminación de la Discriminación contra la Mujer Bolivia. [En línea]. UNHCR - The UN Refugee Agency. 2008. Disponible en: http:// www2.ohchr.org/english/bodies/cedaw/docs/co/ CEDAW.C.BOL.CO.4_sp.pdf.

CIUDADES intermedias. Opinión, Cochabamba, Bolivia, 12 de agosto 2013, p. 7. (En sección: Editorial)

CEPAL Comisión Económica para América Latina. América Latina, proyecciones de población urbana y rural 1970-2025. Boletín Demográfico. (76), 2005.

INE Instituto Nacional de Estadística. Bolivia características de población y vivienda: Censo Nacional de Población y Vivienda 2012. Bolivia, Ministerio de Comunicación. 2012.

--- Censo Nacional de Población y Vivienda 2001.Bolivia. 2001.

LATINOS viven más años y en Bolivia la mortalidad materna aún es elevada. Opinión, Cochabamba, Bolivia, 9 de agosto 2013, p. 10. (En sección: Mundo).

MANZANO Nelson. Estructura económica y competitividad metropolitana en Bolivia. Cochabamba, Kipus. 2010.
PNUD Programa de las Naciones Unidas. El ascenso del Sur: progreso humano en un mundo diverso. Nueva York, PNUD. 2013. ISBN 978-92-1126340-4. Informe sobre desarrollo humano 2013. 